\title{
Expected net present value, expected net future value, and the Ramsey rule
}

\author{
Christian Gollier* \\ Toulouse School of Economics (LERNA and IDEI)
}

June 13, 2009

\begin{abstract}
Weitzman (1998) showed that when future interest rates are uncertain, using the expected net present value implies a term structure of discount rates that is decreasing to the smallest possible interest rate. On the contrary, using the expected net future value criteria implies an increasing term structure of discount rates up to the largest possible interest rate. We reconcile the two approaches by introducing risk aversion and utility maximization. We show that if the aggregate consumption path is optimized and made flexible to news about future interest rates, the two criteria are equivalent. Moreover, they are also equivalent to the Ramsey rule extended to uncertainty.

Keywords: Discount rate, Ramsey rule, climate change, cost-benefit analysis.
\end{abstract}

*This paper benefited from the financial support of the Chair "Sustainable finance and responsible investment" at TSE. The research leading to these results has also received funding from the European Research Council under the European Community's Seventh Framework Programme (FP7/2007-2013) Grant Agreement no. 230589. 


\section{Introduction}

The emergence of the public awareness about the long term sustainability of our growth has been accompanied over the last decade by an important effort from many prominent economists to clarify the way future generations are treated in the standard cost-benefit methodology. Indeed, this methodology is often criticized for sacrificing distant generations. This raises the questions of the level and of the term structure of the discount rate. There is no consensus in our profession about the rate that should be used to discount long term costs and benefits. This implies that economists disagree fundamentally about the intensity of our effort to improve the distant future. This is best illustrated in the field of climate change, but other applications exist in relation to nuclear wastes, biodiversity, genetically modified organisms, or to the preservation of natural resources. Stern (2006), who uses implicitly a discount rate of $1.4 \%$ per year, estimated the current social cost of carbon around 85 dollars per ton of CO2. Nordhaus (2008) criticizes the low discount rate used by Stern, and recommend alternatively a discount rate of $5 \%$, which leads to an estimation of 8 dollars per ton of CO2. This huge difference in the estimation of the social cost of carbon yields radical discrepancies in the policy recommendations related to global changes. This is due to the exponential nature of the impact of a change of the discount rate on net present values (NPV). For example, using a rate of $5 \%$ rather than $1.4 \%$ to discount a cash flow occurring in 200 years reduces its NPV by a factor 1340 .

A standard arbitrage argument justifies using the rate of return of capital, hereafter denoted $\rho$, in the economy as the socially efficient discount rate. Indeed, diverting productive capital to invest in environmental projects with an internal rate of social return below $\rho$ would reduce the welfare of future generations. Another argument is based on the NPV. At equilibrium, the interest rate in the economy equals $\rho$. Suppose that one borrow today at rate $\rho$ an amount equaling to the present value of future cash flows, which implies that these cash flows will be just enough to reimburse the initial loan. This has the effect to transfer all future costs and benefits to today. It implies that the project should be implemented only if its NPV discounted at rate $\rho$ is positive. However, this argument requires to know the returns of capital for the different maturities of the cash flows of the environmental projects under scrutiny. Obviously, they are highly uncertain for the time horizons that we have in mind when we think about climate change or nuclear wastes 
for example. Weitzman $(1998,2001)$ has developed a simple argument based on this fact to recommend a smaller rate to discount distant impacts. The NPV is a decreasing convex function of the interest rate, and the degree of convexity of this function is increasing in the time horizon. Therefore, by Jensen's inequality, introducing an uncertain permanent shock on future interest rates raises the expected NPV, and this effect is increasing in the maturity. It implies that the uncertainty on $\rho$ has an effect on the expected NPV that is equivalent to a sure reduction in $\rho$. It also implies that the term structure of this reduction is increasing. Newel and Pizer (2003) and Groom, Koundouri, Panopoulou and Pantelidis (2007) estimated the impact of the uncertainty about future interest rates on the socially efficient discount rate for different time horizons. More recently, Gollier, Koundouri and Pantelidis (2008) estimated the term structure based on the uncertainty of the GDPweighted world interest rates. They obtained discount rates of $4.2 \%, 2.3 \%$ and $1.8 \%$ respectively for the short term, 100 years and 200 years.

We confront this approach to two other ones. The first alternative approach is exactly symmetric to the one proposed by Weitzman (1998). It measures the impact of the uncertainty on the expected net future value (NFV), as described for example by Gollier (2004), Hepburn and Groom (2007) and Buchholz and Schumacher (2008). Rather than assuming that all costs and benefits of the environmental project are transferred to the present, it assumes that they are all transferred to the terminal date of the project. This alternative approach yields the opposite results: the uncertainty on future interest rates raises the discount rate in an increasing way with the time horizon. A third approach consists in making no intertemporal transfer of costs and benefits, which implies that consumption is modified to compensate them in real time. Under this approach, one needs to compare the impact of uncertain changes in consumption at different dates on the intertemporal welfare. Ramsey (1928) derived a simple formula that equalizes the efficient discount rate net of the rate of pure preference for the present to the product of the growth rate of GDP by the index of aversion to (intertemporal) consumption inequality. Gollier $(2002,2008)$ and Weitzman (2007) extended this formula to take account of the uncertainty on the growth rate of GDP, which is itself linked to the uncertainty of future interest rates. These authors show that the term structure of the efficient discount rate is flat when shocks on the growth rate are temporary, and is decreasing when shocks have a permanent component.

These results are very heterogeneous, and look rather unrelated to each 
others. The aim of this paper is to unify them in an single framework under uncertainty. We show that these approaches are equivalent once risk aversion is properly integrated into the model. We make explicit the investor's objective function, which is assumed to be the standard Discounted Expected Utility criterion. We show how to generalize the discount rates based on the NPV and NFV rules to the case of risk aversion. In fact, we show that the expectations considered above must be based on risk-neutral probabilities, which are proportional to the marginal utility of consumption at the evaluation date. This is in line with the standard consumption-based methodology in finance to estimate risk premia.

Introducing risk aversion alone does not solve the puzzle. Another step must be made, in which the consumption path is optimized. Under the condition that the investor optimizes her consumption plan contingent to the observed interest rate, we show that the NPV and NFV approaches lead to exactly the same term structure of discount rates, which is decreasing and tends to the lowest possible interest rate. Moreover, we show that the two equivalent approaches are then also perfectly compatible with the well-known Ramsey rule.

This paper is mostly non-technical, contrary to Gollier (2009). This companion paper also shows that the decreasing nature of the term structure obtained in this framework depends heavily upon the assumption that shocks are permanent. If they are purely transitory, the term structure of discount rates should be flat. In section 2, we present the discount rates examined respectively by Weitzman (1998) and Gollier (2004) under risk neutrality. Section 3 is devoted to add risk aversion into the picture, and to introduce the Ramsey rule. Finally, we show in section 4 that the three approaches are equivalent once we recognize that agents adapt optimally their consumption plan to news about the future rate of return of capital in the economy. We also illustrate this with a numerical example. 


\section{Net present value and net future value un- der uncertainty}

Consider a simple investment that generates a sure payoff $Z$ at date $t$ per euro invested at date $0 .{ }^{1}$ If $\rho$ is the continuously compounded interest rate during the period, it is optimal to undertake the project if its Net Present Value

$$
N P V=-1+Z e^{-\rho t}
$$

is positive. This NPV rule is sustained by a simple arbitrage argument: implementing the project and borrowing $Z e^{-\rho t}$ at date 0 until date $t$ would generate the sure payoff $N P V$ today, with no other net payoff along the lifetime of the project. Suppose now that the interest rate $\widetilde{\rho}$ that will prevail between dates 0 and $t$ is constant but uncertain. It is unknown at the time the investment decision must be made, but the uncertainty $\widetilde{\rho}$ is fully resolved at date $t=0$. Because the investment opportunity cost is uncertain, it is likely to affect the optimal decision. Weitzman $(1998,2001)$ assumes that the optimal decision criterion in that context is to invest if the expected NPV is positive, i.e., if $-1+Z E e^{-\widetilde{\rho} t}$ is positive. Obviously, this is equivalent to using a discount rate $r_{p}$ such that $e^{-r_{p} t}=E e^{-\widetilde{\rho} t}$, where $r_{p}$ is defined as follows:

$$
r_{p}(t)=-\frac{1}{t} \ln E e^{-\widetilde{\rho} t} .
$$

It is easy to check that $r_{p}(t)$ is less than $E \widetilde{\rho}$, and that it tends to its lowest possible rate for large $t$. The discount rate $r_{p}$ is sustained by a strategy in which the project is implemented and in which the investor borrows at date 0 a random amount $Z e^{-\widetilde{\rho} t}$ at interest rate $\widetilde{\rho}$ until $t$. All the risk is thus borne at date 0 .

One could alternatively consider another arbitrage strategy, in which the investor borrows one euro at date 0 to finance the project. In that case, only one net payoff is generated. It takes place at date $t$ and is equal to the expected Net Future Value $(\mathrm{NFV})-E e^{\widetilde{\rho} t}+Z$. Investing in the project is optimal if the expected NFV is positive. This is equivalent to using a discount rate equaling

$$
r_{f}(t)=\frac{1}{t} \ln E e^{\widetilde{\rho} t}
$$

\footnotetext{
${ }^{1}$ Any sure investment project can be decomposed into a portfolio of investment projects with a single future cash flow occuring at different dates. So this assumption is made without loss of generality.
} 
It is again easy to check that $r_{f}(t)$ is larger than $E \widetilde{\rho}$, and that it tends to its largest possible rate when $t$ tends to infinity. The discount rate $r_{f}$ is sustained by an arbitrage strategy in which the initial investment cost is financed by a loan at rate $\widetilde{\rho}$ between 0 and $t$. All the investment risk is thus borne at the future date $t$ in this case.

In the absence of uncertainty, it is obvious that $r_{f}(t)=r_{p}(t)$, which means that the NPV and NFV criteria are equivalent in that case. This is not the case under uncertainty, as initially observed by Pazner and Razin (1975), and then by Gollier (2004), Hepburn and Groom (2007) and Buchholz and Schumacher (2008). This shows that, in general, the choice of the discount rate cannot be disentangled from how the investment is financed, and from how the risky payoff of the project is allocated through time. Hepburn and Groom (2007) proposed an explanation for the paradox, namely that the certainty-equivalent social discount rate does fall with the passage of time, but it increases as the evaluation date for the investment moves further into the future. In a model with a risk-neutral planner, this provides interesting insights to this paradox, but it leaves us with another unsatisfactory conclusion, namely that the evaluation date is arbitrary and thus one cannot objectively decide which discount rate should be used. They show that the decision criterion is highly sensitive to the arbitrary evaluation date, noticing that "a fuller analysis is required", and concluding that "in the murky waters of intergenerational policy, any theoretical advance providing a ray or two of light is to be welcomed."

This paper is also related to a recent paper by Buchholz and Schumacher (2008), who also recognize the necessity to introduce risk aversion into the analysis. They propose an interesting criterion in which investing at the discount rate $r_{b s}(t)$ is defined in such a way that it yields the same expected utility as investing at the uncertain rate of return of capital: $u\left(\exp r_{b s} t\right)=E u(\exp \tilde{\rho} t)$. They conclude that the discount rate $r_{b s}$ is decreasing or increasing with the time horizon $t$ depending upon the intensity of risk aversion. In particular, a relative risk aversion less than unity yields an increasing term structure. Our approach differs much from Buchholz and Schumacher's one mostly because we use the more standard marginalist approach to asset pricing. 


\section{Three discount rates with risk aversion}

In this section, we introduce risk aversion into the picture. Consider a representative investor with an increasing and concave von Neumann-Morgenstern utility function $u$. In order to determine whether an investment project is socially desirable or not, we define the intertemporal welfare function as follows:

$$
W=\sum_{t=0}^{T} e^{-\delta t} E u\left(\widetilde{c}_{t}\right) .
$$

Parameter $\delta$ is the investor's rate of pure preference for the present, whereas $T$ is the (potentially arbitrarily long) time horizon of the planner. We assume that consumption at date $t$, which is denoted $\widetilde{c}_{t}$, is a random variable. Because the discount factor on utils is exponential, this discounted expected utility model is compatible with a time-consistent behavior. Finally, let $\rho$ denote the rate of return of capital per period. It is a random variable $\widetilde{\rho}$ at the time of the investment decision prior to $t=0$, but the uncertainty is fully revealed at date 0 . Using a standard arbitrage argument, this implies that the socially efficient discount rate ex post is $\rho$. We hereafter determine three different ways to characterize the socially efficient discount rate prior to the realization of $\widetilde{\rho}$.

As in the previous section, consider an investment that generates $Z=e^{r t}$ euros with certainty at date $t$ per euro invested at date 0 , where $r$ is the sure internal rate of return of the project. If $r$ is large enough, the project is socially desirable, i.e., it increases $W$. We define the discount rate associated to maturity $t$ as the critical $r$ such that investing a small amount $\varepsilon$ in the project would have no effect on welfare $W$. However, measuring the impact of the implementation of the investment project on $W$ requires determining first how the benefit of the project is transformed into changes in consumption at different dates.

Let $b$ denote the share of the initial investment cost that is financed through a reallocation of capital in the economy. The remaining share $1-b$ corresponds to a reduction of consumption at date 0 . Thus, $b$ characterizes the way the net benefit is allocated in the lifetime of the project. We assume that at the terminal date $t$ of the project, the borrowed capital and the interest are paid back and reinvested in the economy. It implies that the the net increase in consumption at date $t$ equals $Z-b e^{\rho t}$. Given the financing strategy $b$ which may be made contingent to the realization $\rho$ at date 0 , the 
impact of a marginal investment in the project on the intertemporal social welfare $W$ equals

$$
\Delta W=-E u^{\prime}\left(\widetilde{c}_{0}\right)(1-b)+e^{-\delta t} E u^{\prime}\left(c_{t}\right)\left(e^{r t}-b e^{\widetilde{\rho} t}\right) .
$$

Because $b$ may be contingent to $\widetilde{\rho}$, which may itself be correlated to $\widetilde{c}_{0}$ and $\widetilde{c}_{t}$, this formula cannot be simplified. For each $b$, there is a unique $r$ that solves the optimality condition $\Delta W=0$. We examine three particular specifications for $b$.

A natural candidate for $b$ is $b=0$ : the cash flows of the project are consumed when they occur. In that case, condition $\Delta W=0$ yields the Ramsey discount rate $r=r_{r}(t)$ with

$$
r_{r}(t)=\delta-\frac{1}{t} \ln \frac{E u^{\prime}\left(\widetilde{c}_{t}\right)}{E u^{\prime}\left(\widetilde{c}_{0}\right)} .
$$

For example, assume that relative risk aversion is constant, i.e., $u^{\prime}(c)=c^{-\gamma}$, and that the growth rate of consumption is certain, i.e. $c_{t}=c_{0} e^{g t}$. In that particular case, equation (4) implies that $r_{r}(t)=\delta+\gamma g$, which is the wellknown Ramsey rule: the discount rate net of the rate of impatience equals the product of the growth rate of consumption by the index of relative risk aversion.

An alternative candidate is $b=Z e^{-\rho t}=e^{(r-\rho) t}$. This is the NPV case in which all costs and benefits are transferred to the initial date. Condition $\Delta W=0$ yields $r=r_{p}(t)$, with

$$
r_{p}(t)=-\frac{1}{t} \ln \frac{E u^{\prime}\left(\widetilde{c}_{0}\right) e^{-\widetilde{\rho} t}}{E u^{\prime}\left(\widetilde{c}_{0}\right)} .
$$

This is the "NPV" discount rate under risk aversion. The ratio in the righthand side of this equality is a weighted expectation of $e^{-\widetilde{\rho} t}$. Because the overall risk of the strategy is allocated to consumption at date 0 , the weights are proportional to the marginal utility at that date. In the finance literature, this distortion of objective probabilities is referred to as "risk-neutral probabilities". Notice that the NPV discount rate characterized by equation (5) boils down to the Weitzman discount rate (1) when the representative agent is risk neutral, or when consumption $\widetilde{c}_{0}$ at date 0 is independent of the interest rate $\widetilde{\rho}$. Introducing risk aversion does not change the shape of the term structure, which is decreasing and tends to the smallest possible interest rate for large maturities. 
A third approach consists in assuming that the initial cost of the project is entirely financed by a transfer of capital from other projects $(b=1)$. This leads to the "NFV" discount rate. Equalizing $\Delta W$ to zero for such a $b$ defines the NFV discount rate $r=r_{f}(t)$ :

$$
r_{f}(t)=\frac{1}{t} \ln \frac{E u^{\prime}\left(\widetilde{c}_{t}\right) e^{\widetilde{\rho} t}}{E u^{\prime}\left(\widetilde{c}_{t}\right)} .
$$

This NFV discount rate generalizes the discount rate (2) to the case of risk aversion. The risk-neutral probability distribution weights the objective probabilities to the marginal utility of consumption at date $t$. Without any information about the statistical relationship between $\widetilde{c}_{t}$ and $\widetilde{\rho}$, one cannot characterize the term structure of the NFV discount rate, except in the case of risk neutrality.

As observed by Hepburn and Groom (2007), one could define an infinite number of such discount rates by choosing arbitrarily the evaluation date. The translation of this observation into the framework of this paper is that the socially efficient discount rate depends upon the way the project is financed. This demonstrates that, as suggested by Hepburn and Groom (2007), both Weitzman (1998) and Gollier (2004) are right. Whether one should use a NPV or a NFV approach depends upon who will benefit from the investment.

\section{Unifying field: Optimization of the con- sumption strategy}

The conclusion of the previous section is problematic. Standard cost-benefit analysis states that the way one evaluates an investment project should not depend upon the way the project is financed. There should be only one term structure of discount rates. This property is one of the cornerstones of public finance. In this section, we show that the three discount rates $r_{r}$, $r_{p}$ and $r_{f}$ are in fact all equal if we recognize that economic agents optimize their consumption path.

From date 0 on, the investor knows the rate of return on capital $\rho$. Reducing consumption by $\varepsilon$ at date $t_{1}$ allows the agent to increase consumption by $\varepsilon e^{\rho\left(t_{2}-t_{1}\right)}$ at date $t_{2}$. Therefore, the optimal consumption path conditional to $\rho$ must be such that $u^{\prime}\left(c_{t_{1}}\right)$ equals $e^{(\rho-\delta)\left(t_{2}-t_{1}\right)} u^{\prime}\left(c_{t_{2}}\right)$, otherwise transferring consumption from one date to the other would increase the intertemporal 
welfare $W$. This implies in particular that conditional to $\rho$, we must have that

$$
u^{\prime}\left(c_{0}\right)=e^{(\rho-\delta) t} u^{\prime}\left(c_{t}\right) .
$$

Observe that it implies that optimal consumption plans in general depend upon $\rho$.

This optimality condition implies that $r_{r}(t)=r_{p}(t)=r_{f}(t)$, and more generally that the socially efficient discount rate is independent upon the evaluation date, or upon the way (marginal) projects are financed. Indeed, using condition (7), we have that

$$
r_{r}(t)=\delta-\frac{1}{t} \ln \frac{E u^{\prime}\left(\widetilde{c}_{t}\right)}{E u^{\prime}\left(\widetilde{c}_{0}\right)}=-\frac{1}{t} \ln \frac{E u^{\prime}\left(\widetilde{c}_{0}\right) e^{-\widetilde{\rho} t}}{E u^{\prime}\left(\widetilde{c}_{0}\right)}=r_{p}(t),
$$

and

$$
r_{f}(t)=\frac{1}{t} \ln \frac{E u^{\prime}\left(\widetilde{c}_{t}\right) e^{\widetilde{\rho} t}}{E u^{\prime}\left(\widetilde{c}_{t}\right)}=-\frac{1}{t} \ln \frac{E u^{\prime}\left(\widetilde{c}_{0}\right) e^{-\widetilde{\rho} t}}{E u^{\prime}\left(\widetilde{c}_{0}\right)}=r_{p}(t) .
$$

Proposition 1 (Equivalence Property) Under the assumption that consumers can make their consumption plan flexible to changes in the return of capital, the socially efficient discount rate is independent of how the uncertain net benefit of the environmental project is allocated through time. It means in particular that $r_{r}(t)=r_{p}(t)=r_{f}(t)$.

This is in fact an application of the envelop theorem. Thus, we have reconciled the various approaches that have recently been used to discuss whether the discount rate should be decreasing with the time horizon. Under this framework in which the shock on interest rates is permanent, the socially efficient discount rate is decreasing and tends to the smallest possible interest rate.

In order to illustrate these properties, let us consider an economy in which the infinitely lived representative agent with a unit initial wealth has a rate of impatience equaling $\delta=2 \%$ and an index of relative risk aversion equaling $\gamma=2$. It implies that, conditional to $\rho$, consumption $c_{t}$ equals $(\rho-g) \exp (g t)$, where $g=(\rho-\delta) / \gamma$ is the rate of growth of consumption. ${ }^{2}$ Suppose first that the rate of return of capital is risk free and equal to $\rho=5 \%$. In that case,

\footnotetext{
${ }^{2}$ The condition $g<\rho$ is necessary and sufficient for the existence of a solution to the consumption problem with an infinite horizon. Condition $\gamma>1$ guarantees that this condition holds.
} 


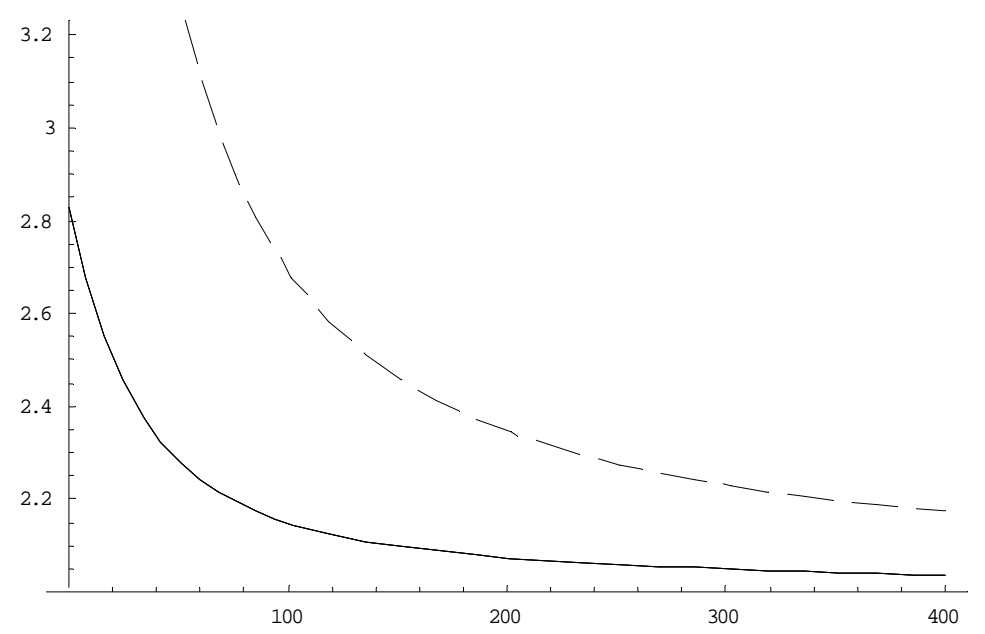

Figure 1: The term structure of discount rates (in $\%$ per year) when $\delta=2 \%$, $\gamma=2$, and $\rho$ equals $2 \%$ or $8 \%$ with equal probabilities. This curve is obtained by using any of the 3 formulas (4), (5) or (6). The dashed curve correspond to the NPV formula (1) proposed by Weitzman (1998).

the initial consumption level is $c_{0}=0.035$, and the growth of consumption is $g=1.5 \%$. Of course, the efficient discount rate is a flat $r_{r}(t)=r_{p}(t)=$ $r_{f}(t)=5 \%$.

Suppose alternatively that the rate of return of capital in the economy will be either $2 \%$ or $8 \%$ with equal probabilities. If one observes the low rate of return $\rho=2 \%$ at date 0 , the planner should select a flat consumption plan $c_{t}=0.02$. On the contrary, if one observes the high rate of return $\rho=8 \%$, the planner should select $c_{0}=0.05$ and $g=3 \%$. Using any of the three formulas (4), (5) or (6) yields a term structure of the efficient discount rates ex ante that is described in Figure 1. Observe that the discount rate for short horizons is around $2.8 \%$, which is far below the efficient discount rate of $5 \%$ that would prevail without uncertainty. The effect of uncertainty is magnified for larger time horizons. The discount rate tends to its smallest possible value $(2 \%)$ when $t$ tends to infinity. We also depicted in this graph the term structure that is recommended by Weitzman (1998) (equation (1)) in this context. We see that the effect of uncertainty is underestimated when using the Weitzman's equation (1), but that the shapes of the term structure are similar. 
Now, let us alternatively examine a situation in which the three discount rates defined in section 3 are different because consumption plans are not optimized. Observe that the realization of the uncertainty at date 0 forces the representative agent to a huge change in initial consumption, which is $150 \%$ higher in the good state than in the bad state. For many reasons, it seems quite unrealistic to believe that our society has such a degree of flexibility and reactivity. Suppose alternatively that initial wealth is not sensitive to news on capital productivity. Suppose more specifically that $c_{0}=0.035$ in the two states as in the certainty case. Suppose also that the planner fixes the constant growth rate of consumption in each state that is compatible with the intertemporal budget constraint. This implies that the growth rate $g$ is equal to $-1.5 \%$ or $4.5 \%$ respectively in the bad state and in the good state. These plans are obviously inefficient since they fail to satisfy the firstorder condition (7). In that case, the efficient discount rate depends upon the way projects are financed. Figure 2 describes the term structure of the discount rate respectively when no transfer of cash flows is made $\left(r_{r}(t)\right)$, when all cash flows are transferred to the present $\left(r_{p}(t)\right)$, or when all cash flows are transferred to the terminal date of the investment $\left(r_{f}(t)\right)$. Interestingly enough, the Weitzman's NPV approach is the one for which the effect of uncertainty is the smallest, for all maturities.

\section{Conclusion}

We have shown that the choice of the discount rate for marginal projects depends in general upon how the benefits and costs of the project are allocated through time. This explains the differences between the approaches examined respectively by Ramsey (1928), Weitzman (1998) and Gollier (2004). The Ramsey rule is obtained by assuming that the cash flows of the investment are consumed at the time at which they occur. In Weitzman's NPV approach, it is assumed that all costs and benefits are consumed at date 0 , whereas they are all consumed at the terminal date in Gollier's NFV approach. Of course, the risk associated to the various financing strategies must be taken into account given the risk aversion of the representative agent. This must be done by using risk-neutral probability distributions that vary with the evaluation date.

Our main contribution is to show that these different approaches to discounting are completely equivalent once we recognize that consumers react 


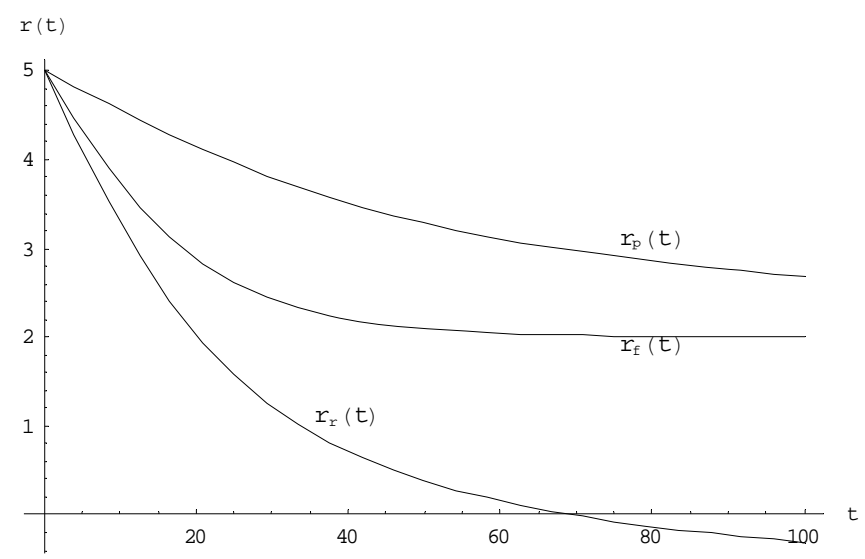

Figure 2: The term structure of discount rates when consumption plans are inefficient. The parameters of the model are as in Figure 1, but we assume here that $c_{0}=0.035$, and that $g$ equals $-1.5 \%$ and $+4.5 \%$ respectively in the bad state and in the good state.

optimally to changes in the interest rate. Ramsey, Weitzman and Gollier's approaches lead to the same term structure of discount rates when consumption paths are optimal. When shocks on future interest rates have a permanent component, the discount rate has a decreasing term structure. It tends to the smallest possible rate for very distant time horizons. When applied to climate change, this conclusion tends to go in favor of the low implicit discount rate used in the Stern Review (2006), and therefore in favor of a relatively large price for carbon.

When consumption plans cannot be fully optimized and made flexible to news, the way the benefits of the project are allocated into changes in consumption will not be neutral when evaluating the project. It will affect the discount rate that should be used to evaluate environmental projects. It is hard to believe that our collective consumption trajectory that span many different generations over the next few centuries is socially efficient given the uncertainty we face today. Obvious conflicts of interest and rigidities inhibit our economies to adapt rapidly to news about the future productivity of capital and about the future growth of the economy. Thus, we conclude that one cannot separate the problem of the choice of the rate to discount the cash flows of an environmental project from the question of how it will be financed and of who will cash the benefits or pay the costs. 


\section{Bibliography}

Buchholz, W., and J. Schumacher, (2008), Discounting the long distant future: Simple explanation for the Weitzman-Gollierpuzzle, mimeo.

Gollier, C., (2002), Discounting an uncertain future, Journal of Public Economics, 85, 149-166.

Gollier, C., (2004), Maximizing the expected net future value as an alternative strategy to gamma discounting, Finance Research Letters, 1, 85-89.

Gollier, C., (2008), Discounting with fat-tailed economic growth, Journal of Risk and Uncertainty, 37, 171-186.

Gollier, C., (2009), Should We Discount the Far-Distant Future at Its Lowest Possible Rate?, Economics: The Open-Access, Open-Assessment E-Journal, Vol. 3, 2009-25.

Gollier, C., P.Koundouri, and T. Pantelidis, (2008), Declining discount rates: Economic justifications and implications for long-run policy, Economic Policy (56), 757-795.

Groom, B., P. Koundouri, E. Panopoulou, and T. Pantelidis, (2007), An econometric approach to estimating long-run discount rates, Journal of Applied Econometrics, 22, 641- 656.

Hepburn, C., and B. Groom, (2007), Gamma discounting and expected net future value, Journal of Environmental Economics and Management (53), 99-109.

Newell, R., and W. Pizer, (2003), Discounting the benefits of climate change mitigation: How much do uncertain rates increase valuations?, Journal of Environmental Economics and Management, 46(1), 52-71.

Nordhaus, W., (2008), A question of balance: Weighing the options on global warming policies, Yale University Press, New Haven and London.

Pazner, E.A., and A. Razin, (1975), On expected present value vs. expected future value, Journal of Finance, (30), 875-877. 
Ramsey, F.P., (1928), A mathematical theory of savings, The Economic Journal, 38, 543-59.

Stern, N., (2006), The Economics of Climate Change: The Stern Review, Cambridge University Press, Cambridge.

Weitzman, M.L., (1998), Why the far-distant future should be discounted at its lowest possible rate?, Journal of Environmental Economics and Management, 36, 201-208.

Weitzman, M.L., (2001), Gamma discounting, American Economic Review, 91, 260-271.

Weitzman, M.L., (2007), Subjective expectations and asset-return puzzle, American Economic Review, 97, 1102-1130. 